\title{
OPEN Effect of data leakage in brain MRI classification using 2D convolutional neural networks
}

\author{
Ekin Yagis $^{1,5}$, Selamawet Workalemahu Atnafu ${ }^{2,5}$, Alba García Seco de Herrera ${ }^{1,6}$, \\ Chiara Marzi ${ }^{2}$, Riccardo Scheda ${ }^{2}$, Marco Giannelli ${ }^{3}$, Carlo Tessa ${ }^{4}$, Luca Citi ${ }^{1,6}$ \& \\ Stefano Diciotti ${ }^{2,6 \bowtie}$
}

In recent years, 2D convolutional neural networks (CNNs) have been extensively used to diagnose neurological diseases from magnetic resonance imaging (MRI) data due to their potential to discern subtle and intricate patterns. Despite the high performances reported in numerous studies, developing CNN models with good generalization abilities is still a challenging task due to possible data leakage introduced during cross-validation (CV). In this study, we quantitatively assessed the effect of a data leakage caused by 3D MRI data splitting based on a 2D slice-level using three 2D CNN models to classify patients with Alzheimer's disease (AD) and Parkinson's disease (PD). Our experiments showed that slice-level CV erroneously boosted the average slice level accuracy on the test set by $30 \%$ on Open Access Series of Imaging Studies (OASIS), $29 \%$ on Alzheimer's Disease Neuroimaging Initiative (ADNI), 48\% on Parkinson's Progression Markers Initiative (PPMI) and 55\% on a local de-novo PD Versilia dataset. Further tests on a randomly labeled OASIS-derived dataset produced about $96 \%$ of (erroneous) accuracy (slice-level split) and $50 \%$ accuracy (subject-level split), as expected from a randomized experiment. Overall, the extent of the effect of an erroneous slice-based $\mathrm{CV}$ is severe, especially for small datasets.

Deep learning has become a popular class of machine learning algorithms in computer vision and has been successfully employed in various tasks, including multimedia analysis (image, video, and audio analysis), natural language processing, and robotics ${ }^{1}$. In particular, deep convolutional neural networks (CNNs) hierarchically learn high-level and complex features from input data, hence eliminating the need for handcrafting features, as in the case of conventional machine learning schemes ${ }^{2}$.

The application of these methods in neuroimaging is rapidly growing (see Greenspan et al. ${ }^{3}$ and Zaharchuk et al. ${ }^{4}$ for reviews). Several studies employed deep learning methods for image improvement and transformation $^{5-10}$. Other studies performed lesion detection and segmentation ${ }^{11-13}$ and image-based diagnosis using different CNNs architectures ${ }^{14,15}$. Deep learning has also been applied to more complex tasks, including identifying patterns of disease subtypes, determining risk factors, and predicting disease progression (see, e.g., Zaharchuk et al. ${ }^{4}$ and Davatzikos ${ }^{16}$ for reviews). Early works applied stacked auto encoders ${ }^{14,17,18}$ and deep belief networks ${ }^{19}$ to classify neurological patients from healthy subjects using data collected from different neuroimaging modalities, including magnetic resonance imaging (MRI), positron emission tomography (PET), resting-state functional MRI (rsfMRI), and the combination of these modalities ${ }^{20}$

Some authors reported very high accuracies in classifying patients with neurological diseases, such as Alzheimer's disease (AD) and Parkinson's disease (PD). For a binary classification of AD vs. healthy controls, Hon and $\mathrm{Khan}^{21}$ reported accuracy up to $96.25 \%$ using a transfer learning strategy. Sarraf et al. ${ }^{22}$ classified subjects as $\mathrm{AD}$ or healthy controls with a subject-level accuracy of $100 \%$ by adopting LeNet- 5 and GoogleNet network architectures. In other studies, CNNs have been used for performing multi-class discrimination of subjects. Recently, Wu et al. ${ }^{23}$ adopted a pre-trained CaffeNet and achieved accuracy of $98.71 \%, 72.04 \%$, and $92.35 \%$ for

\footnotetext{
${ }^{1}$ School of Computer Science and Electronic Engineering, University of Essex, Colchester, UK. ${ }^{2}$ Department of Electrical, Electronic, and Information Engineering "Guglielmo Marconi", University of Bologna, Via dell'Università 50, 47521 Cesena, Italy. ${ }^{3}$ Unit of Medical Physics, Pisa University Hospital "Azienda Ospedaliero-Universitaria Pisana", Pisa, Italy. "Division of Radiology, Versilia Hospital, Azienda USL Toscana Nord Ovest, Lido di Camaiore, LU, Italy. ${ }^{5}$ These authors contributed equally: Ekin Yagis and Selamawet Workalemahu Atnafu. ${ }^{6}$ These authors jointly supervised this work: Alba García Seco de Herrera, Luca Citi and Stefano Diciotti. ${ }^{\square}$ email: stefano.diciotti@ unibo.it
} 


\begin{tabular}{|c|c|c|c|c|c|c|}
\hline Disorder & References & Groups (number of subjects) & Machine learning model & Data split method & Type of data leakage & Accuracy (\%) \\
\hline \multirow{9}{*}{$\mathrm{AD} / \mathrm{MCI}$} & Gunawardena et al. ${ }^{36}$ & AD-MCI-HC (36) & 2D CNN & 4:1 train/test slice-level split & Wrong split & 96.00 \\
\hline & Hon and Khan ${ }^{21}$ & AD-HC (200) & 2D CNN (VGG16) & 4:1 train/test slice-level split & Wrong split & 96.25 \\
\hline & Jain et al..$^{37}$ & AD-MCI-HC (150) & 2D CNN (VGG16) & 4:1 train/test slice-level split & Late and wrong split & 95.00 \\
\hline & Khagi et al. ${ }^{38}$ & AD-HC (56) & $\begin{array}{l}\text { 2D CNN (AlexNet, } \\
\text { GoogLeNet,ResNet50, new } \\
\text { CNN) }\end{array}$ & $\begin{array}{l}\text { 6:2:2 train/validation/test } \\
\text { slice-level split }\end{array}$ & Wrong split & 98.00 \\
\hline & Sarraf et al..$^{22}$ & AD-HC (43) & 2D CNN (LeNet-5) & $\begin{array}{l}\text { 3:1:1 train/validation/test } \\
\text { slice-level split }\end{array}$ & Wrong split & 96.85 \\
\hline & Wang et al. ${ }^{39}$ & MCI-HC (629) & $2 \mathrm{D} C N N$ & $\begin{array}{l}\text { Data augmentation }+10: 3: 3 \\
\text { train/validation/test split by } \\
\text { MRI slices } \\
\end{array}$ & $\begin{array}{l}\text { Wrong split and augmenta- } \\
\text { tion before split }\end{array}$ & 90.60 \\
\hline & Puranik et al. ${ }^{40}$ & AD/EMCI-HC (75) & $2 \mathrm{D} \mathrm{CNN}$ & $\begin{array}{l}\text { 17:3 train/test split by MRI } \\
\text { slices }\end{array}$ & Wrong split & 98.40 \\
\hline & Basheera et al. ${ }^{41}$ & AD-MCI-HC (1820) & $2 \mathrm{D} \mathrm{CNN}$ & $\begin{array}{l}\text { 4:1 train/test split by MRI } \\
\text { slices }\end{array}$ & Wrong split & 90.47 \\
\hline & Nawaz et al..$^{42}$ & AD-MCI-HC (1726) & $2 \mathrm{D} \mathrm{CNN}$ & 6:2:2 slice level split & Wrong split & 99.89 \\
\hline
\end{tabular}

Table 1. Summary of the previous studies performing classification of neurological disorders using MRI and with clear data leakage (see also Supplementary Table S1 for a detailed description). AD Alzheimer's disease, $H C$ healthy controls, $M C I$ mild cognitive impairment.

a three-way classification between healthy controls, stable mild cognitive impairment (MCI), and progressive MCI patients, respectively. In another work by Islam and Zhang ${ }^{24}$, an ensemble system of three homogeneous CNNs has been proposed, and average multi-class classification accuracy of $93.18 \%$ was found on the Open Access Series of Imaging Studies (OASIS) dataset. For the classification of PD, Esmaeilzadeh et al. ${ }^{25}$ classified PD patients from healthy controls based on MRI and demographic information (i.e., age and gender). With the proposed 3D model, they achieved 100\% accuracy on the test set. In another study by Sivaranjini and Sujatha ${ }^{26}$, a pre-trained 2D CNN AlexNet architecture was used to classify PD patients vs. healthy controls, resulting in an accuracy of $88.9 \%$.

Although excellent performances have been shown by using deep learning for the classification of neurological disorders, there are still many challenges that need to be addressed, including complexity and difficulty in interpreting the results due to highly nonlinear computations, non-reproducibility of the results, and data/ information and, especially, data overfitting (see Vieira et al. ${ }^{20}$ and Davatzikos ${ }^{16}$ for reviews).

Overly optimistic results may be due to data leakage-a process caused by the use of information in the model training that is not expected to be available at prediction time. See Kaufman et al. ${ }^{27}$ for further details on a formal definition of data leakage. Data leakage can be due to a target (class label) leakage or incorrect data split. For example, data leakage may occur when feature selection is performed based on the whole dataset before crossvalidation $^{28,29}$. In this case, the target variable of samples in the test sets may be erroneously used for improving the learning process. Several cases may be related to an incorrect data split. For example, when the data augmentation step is performed before dividing the test set from the training data (late split), the augmented data generated from the same original image can be seen in both training and test data, leading to incorrect inflated performance ${ }^{30}$. Another form of train-test contamination that leads to data leakage is when the same test set is used to optimize the training hyperparameters and evaluate the model performance ${ }^{29}$. A different use of information not available at prediction time occurs using longitudinal data, when there is a danger of information leaking from the future to the past. A particularly insidious form of data leakage may occur when information about the target inadvertently leaks into the input data, for example the presence of a ruler, markings or treatment devices in a medical image may correlate with the class label ${ }^{31-33}$.

While concluding that data leakage leads to overly optimistic results will surprise few practitioners, we believe that the extent to which this is happening in neuroimaging applications is mostly unknown, especially in small datasets. As we completed this study, we became aware of independent research by Wen et al. ${ }^{30}$ that corroborates part of our conclusions regarding the problem of data leakage. They successfully suggested a framework for the reproducible assessment of AD classification methods. However, the architectures have not been trained and tested on smaller datasets typical of clinical practice, and they mainly employed hold-out model validation strategies rather than cross-validation $(\mathrm{CV})$ - that gives a better indication of how well a model performs on unseen data ${ }^{34,35}$. Moreover, the authors focused on illustrating the effect of data leakage on the classification of $\mathrm{AD}$ patients only.

Unfortunately, the problem of data leakage incurred by incorrect data split is not only limited within the area of $\mathrm{AD}$ classification but can also be seen in various other neurological disorders. It is more common to observe the data leakage in $2 \mathrm{D}$ architectures, yet some forms of data leakage, such as late split, could be present in 3D $\mathrm{CNN}$ studies as well. Moreover, although deep complex classifiers are more prone to overfitting, also conventional machine learning algorithms may be affected by data leakage. A summary of these works with clear and potential data leakage is given in Tables 1 and 2, respectively. Other works with insufficient information to assess data leakage are reported in Table 3.

In this study, we addressed the issue of data leakage in one of the most common classes of deep learning models, i.e., 2D CNNs, caused by incorrect dataset split of 3D MRI data. Specifically, we quantified the effect of data leakage on $\mathrm{CNN}$ models trained on different datasets of $\mathrm{T}_{1}$-weighted brain $\mathrm{MRI}$ of healthy controls and patients 


\begin{tabular}{|c|c|c|c|c|c|c|}
\hline Disorder & References & Groups (number of subjects) & Machine learning model & Data split method & Type of data leakage & Accuracy (\%) \\
\hline \multirow{4}{*}{$\mathrm{AD} / \mathrm{MCI}$} & Farooq et al. ${ }^{43}$ & AD-MCI-LMCI-HC (355) & $\begin{array}{l}\text { 2D CNN (GoogLeNet and } \\
\text { modified ResNet) }\end{array}$ & $\begin{array}{l}3: 1 \text { train/test (potential) slice- } \\
\text { level split }\end{array}$ & Wrong split & 98.80 \\
\hline & Ramzan et al. ${ }^{44}$ & $\begin{array}{l}\text { HC-SMC- EMCI- } \\
\text { MCI-LMCI-AD (138) }\end{array}$ & 2D CNN (ResNet-18) & $\begin{array}{l}\text { 7:2:1 train/validation/test } \\
\text { (potential) slice-level split }\end{array}$ & Wrong split & 100 \\
\hline & Raza et al. ${ }^{45}$ & AD-HC (432) & 2D CNN (AlexNet) & $\begin{array}{l}\text { 4:1 train/test (potential) slice- } \\
\text { level split }\end{array}$ & Wrong split & 98.74 \\
\hline & Pathak et al. ${ }^{46}$ & AD-HC (266) & $2 \mathrm{D} \mathrm{CNN}$ & 3:1 (potential) slice level split & Wrong split & 91.75 \\
\hline \multirow{2}{*}{ ASD } & Libero et al. ${ }^{47}$ & ASD-TD (37) & Decision tree & unclear & $\begin{array}{l}\text { Entire data set used for } \\
\text { feature selection }\end{array}$ & 91.90 \\
\hline & Zhou et al. ${ }^{48}$ & ASD-TD/HC (280) & Random tree classifier & $4: 1$ train/test split & $\begin{array}{l}\text { Entire data set used for } \\
\text { feature selection }\end{array}$ & 100 \\
\hline $\mathrm{PD}$ & Sivaranjini, et al. ${ }^{26}$ & PD-HC (182) & $2 \mathrm{D} \mathrm{CNN}$ & $\begin{array}{l}4: 1 \text { train/test split by MRI } \\
\text { slices }\end{array}$ & Wrong split & 88.90 \\
\hline TBI & Lui et al. ${ }^{49}$ & TBI-HC (47) & Multilayer perceptron & tenfold CV & $\begin{array}{l}\text { Entire data set used for } \\
\text { feature selection }\end{array}$ & 86.00 \\
\hline Brain tumor & Hasan et al. ${ }^{50}$ & Tumor-HC (600) & $\mathrm{MGLCM}+2 \mathrm{D} C \mathrm{CNN}+\mathrm{SVM}$ & tenfold CV & $\begin{array}{l}\text { Wrong split and entire data } \\
\text { set used for feature selection }\end{array}$ & 99.30 \\
\hline
\end{tabular}

Table 2. Summary of the previous studies performing classification of neurological disorders using MRI and suspected to have potential data leakage (see also Supplementary Table S2 for a detailed description). $A D$ Alzheimer's disease, $A S D$ Autism spectrum disorder, $E M C I$ early mild cognitive impairment, $H C$ healthy controls, $L M C I$ late mild cognitive impairment, $M C I$ Mild cognitive impairment, MGLCM modified gray level co-occurrence matrix, PD Parkinson's disease, SMC subjective memory concerns, TBI traumatic brain injury, TD typically developing.

\begin{tabular}{|l|l|l|l|l|l|}
\hline Disorder & References & $\begin{array}{l}\text { Groups (number of } \\
\text { subjects) }\end{array}$ & Machine learning model & Data split method & Accuracy (\%) \\
\hline \multirow{2}{*}{ AD/MCI } & Al-Khuzaie et al. ${ }^{51}$ & AD-HC $(240)$ & 2D CNN & (Potential) slice-level split & 99.30 \\
\cline { 2 - 6 } & Wu et al. ${ }^{23}$ & AD-HC $(457)$ & $2 \mathrm{D} \mathrm{CNN}$ & $\begin{array}{l}\text { Data augmentation }+2: 1 \\
\text { train/test split by MRI slices }\end{array}$ & 97.58 \\
\hline
\end{tabular}

Table 3. Summary of the previous studies performing classification of neurological disorders using MRI and that provide insufficient information to assess data leakage (see also Supplementary Table S3 for a detailed description). AD Alzheimer's disease, $H C$ healthy controls, $M C I$ mild cognitive impairment.

with neurological disorders using a nested CV scheme with two different data split strategies: (a) subject-level split, avoiding any form of data leakage and (b) slice-level split, in which different slices of the same subject are contained both in the training and the test folds (thus data leakage will occur). We focused our attention on both large (about 200 subjects) and small (about 30 subjects) datasets to evaluate a possible increase of performance overestimation when a smaller dataset was used, as is often the case in clinical practice. This paper expands on the preliminary results by Yagis et al..$^{52}$, offering a broader investigation on the issue. In particular, we performed the classification of AD patients using the following datasets: (1) OASIS-200, consisting of randomly sampled $100 \mathrm{AD}$ patients and 100 healthy controls from the OASIS-1 study ${ }^{53}$, (2) ADNI, including $100 \mathrm{AD}$ patients and 100 healthy controls randomly sampled from Alzheimer's Disease Neuroimaging Initiative (ADNI) ${ }^{54}$, and (3) OASIS-34, composed of 34 subjects (17 AD patients and 17 healthy controls) randomly selected from the OASIS-200 dataset. Given that the performance of a model trained on a small sample dataset could depend on the selected samples, we created ten instances of the OASIS-34 dataset by randomly sampling from the OASIS-200 dataset ten times independently. The subject IDs included in each instance are found in Supplementary Table S4. Moreover, we generated a different dataset, called OASIS-random, where, for each subject of the OASIS-200 dataset, a fake random label of either AD patient or healthy control was assigned. In this case, the image data had no relationship with the assigned labels. Besides, we included two $\mathrm{T}_{1}$-weighted images datasets of patients with de-novo PD: PPMI, including 100 de-novo PD patients and 100 healthy controls randomly chosen from the public Parkinson's Progression Markers Initiative (PPMI) dataset ${ }^{55}$, and Versilia, a small-sized private clinical dataset of 17 patients with de-novo PD and 17 healthy controls. A detailed description of each dataset has been reported in the "Materials and methods" section.

\section{Results}

For AD classification, accuracy on the test set, using subject-level CV, was below $71 \%$ for large datasets (OASIS200 and ADNI), whereas they were below 59\% for smaller datasets (OASIS-34). Regarding de novo PD classification, they were around 50\% for both large (PPMI) and small (Versilia) datasets. Conversely, slice-level CV erroneously produced very high classification accuracies on the test set in all datasets (higher than $94 \%$ and $92 \%$ on large and small datasets, respectively), leading to deceptive, over-optimistic results (Table 4). 


\begin{tabular}{|c|c|c|c|c|c|c|}
\hline \multirow[b]{2}{*}{ Dataset } & \multirow[b]{2}{*}{ Network architecture } & \multicolumn{2}{|c|}{ Training set accuracy (\%) } & \multicolumn{3}{|c|}{ Test set accuracy (\%) } \\
\hline & & Subject-level split & Slice-level split & Subject-level split & Slice-level split & Difference \\
\hline \multirow{3}{*}{ OASIS-200 } & VGG16-v1 & 95.93 & 99.85 & 66.0 & 94.18 & 28.18 \\
\hline & VGG16-v2 & 95.13 & 100 & 66.13 & 96.99 & 30.86 \\
\hline & ResNet-18 & 100 & 100 & 68.87 & 98.96 & 30.1 \\
\hline \multirow{3}{*}{ OASIS-34 } & VGG16-v1 & 88.94 & 100 & 54.35 & 99.19 & 44.84 \\
\hline & VGG16-v2 & 96.94 & 100 & 54.34 & 99.33 & 44.99 \\
\hline & ResNet-18 & 100 & 100 & 57.49 & 98.96 & 41.47 \\
\hline \multirow{3}{*}{ OASIS-random } & VGG16-v1 & 63.38 & 100 & 53.37 & 95.93 & 42.56 \\
\hline & VGG16-v2 & 69.17 & 100 & 49.25 & 94.81 & 45.56 \\
\hline & ResNet-18 & 84.49 & 99.09 & 50.8 & 93.74 & 42.94 \\
\hline \multirow{3}{*}{ ADNI } & VGG16-v1 & 91.09 & 100 & 70.12 & 95.31 & 25.19 \\
\hline & VGG16-v2 & 80.49 & 100 & 66.49 & 95.24 & 28.75 \\
\hline & ResNet-18 & 100 & 100 & 68.68 & 96.87 & 30.19 \\
\hline \multirow{3}{*}{ PPMI } & VGG16-v1 & 76.8 & 100 & 48.24 & 93.99 & 45.75 \\
\hline & VGG16-v2 & 73.19 & 100 & 46.93 & 94.37 & 47.44 \\
\hline & ResNet-18 & 100 & 100 & 48.06 & 96.12 & 44.06 \\
\hline \multirow{3}{*}{ Versilia } & VGG16-v1 & 99.72 & 100 & 53.86 & 95.97 & 42.11 \\
\hline & VGG16-v2 & 76.89 & 100 & 42.97 & 97.8 & 54.83 \\
\hline & ResNet-18 & 99.90 & 95.13 & 51.36 & 92.63 & 41.27 \\
\hline
\end{tabular}

Table 4. Mean slice-level accuracy on the training and test set of the outer CV over fivefold nested CV has been reported for three 2D CNN models (see "Materials and methods" section), all datasets, and two data split methods (slice-level and subject-level). The difference between accuracy using slice-level and subject-level split in the test set has also been reported.

The worst-case stemmed from the randomly labeled OASIS dataset, which resulted in a model with unacceptably high performances (accuracy on the test set more than 93\%) using slice-level CV, whereas classification results obtained using a subject-level CV were about $50 \%$, in accordance with the expected outcomes for a balanced dataset with completely random labels.

\section{Discussion}

In this study, we quantitatively assessed the extent of the overestimation of the model's classification performance caused by an incorrect slice-level CV, which is unfortunately adopted in neuroimaging literature (see Tables 1, 2, 3). More specifically, we showed the performance of three 2D CNN models (two VGG variants and one ResNet-18, see "Materials and methods" section) trained with subject-level and slice-level CV data splits to classify $\mathrm{AD}$ and $\mathrm{PD}$ patients from healthy controls using $\mathrm{T}_{1}$-weighted brain MRI data. Our results revealed that pooling slices of MRI volumes for all subjects and then dividing randomly into training and test set leads to significantly inflated accuracies (in some cases from barely above chance level to about 99\%). In particular, slice-level CV erroneously increased the average slice level accuracy on the test set by $40-55 \%$ on smaller datasets (OASIS-34 and Versilia) and 25-45\% on larger datasets (OASIS-200, ADNI, PPMI). Moreover, we also conducted an additional experiment in which all the labels of the subjects were fully randomized (OASIS-random dataset). Even under such circumstances, using the slice-level split, we achieved an erroneous $95 \%$ classification accuracy on the test set with all models, whereas we found $50 \%$ accuracy using a subject-level data split, as expected from a randomized experiment. This large (and erroneous) increase in performance could be due to the high intrasubject correlation among $\mathrm{T}_{1}$-weighted slices, resulting in a similar information content present in slices of the same subject ${ }^{56}$.

In AD classification, three previous studies ${ }^{21,22,43}$, using similar deep networks (VGG16, ResNet-18 and LeNet5 , respectively), reported higher classification accuracies $(92.3 \%, 98.0 \%$ and $96.8 \%$, respectively) than ours. However, there is a strong indication that these performances are massively overestimated due to a slice-level split. In particular, in one of these works ${ }^{21}$, the presence of data leakage was further corroborated by the source code accompanying the paper and confirmed by our data. In fact, when we used the same dataset of Hon and $\mathrm{Khan}^{21}$ (OASIS-200 dataset), our VGG16 models achieved only 66\% classification accuracy with subject-level split, whereas they boosted to about $97 \%$ with a slice-level split. Similar findings were presented by Wen et al..$^{30}$, who used an ADNI dataset with 330 healthy controls and $336 \mathrm{AD}$ patients. Indeed, using baseline data, they reported a $79 \%$ of balanced accuracy in the validation set with a subject-level split which increased up to $100 \%$ with a slice-level split.

One of the main issues in the classification of neurological disorders using deep learning is data scarcity ${ }^{57}$. Not only because labeling is expensive but also because privacy reasons and institutional policies make acquiring and sharing large sets of labeled imaging data even more challenging ${ }^{58}$. To show the impact of data size on model performance, we created 10 small subsets from the OASIS dataset (OASIS-34 datasets). As expected, when we reduced the data, we obtained lower classification accuracies with all the networks using the subject-level data split method. However, when the slice-level method was used, the models erroneous achieved better results on 
OASIS-34 than on the OASIS-200 dataset. Similarly, models trained on the Versilia dataset (34 subjects) produced inflated results with the slice-level split. Overall, these results indicate that data leakage is highly relevant, especially when small datasets are used, which may, unfortunately, be common in clinical practice.

It is well-known that data leakage leads to inflating performance-and this phenomenon is not specific to brain MRI or deep learning, but it can occur in any machine learning system. Nevertheless, the degree of overestimation quantified through our experiments was surprising. Unfortunately, in the literature, the precise application of CV is frequently not well-documented, and the source code is not available, although we have observed these issues mostly in manuscripts that were either not peer-reviewed or not rigorously peer-reviewed (see Tables 1, 2, 3). Overall, this situation leaves the neuroimaging community unable to trust the (sometimes) promising results published. Regardless of the network architecture, the number of subjects, and the level of complexity of the classification problem, all experiments that applied slice-level CV yielded very high classification accuracies on the test set as a result of incorporating different slices of the same subject in both the training and test sets. Considering classifications on 2D MRI images, we showed that it is crucial that the CV split be done based on the subject-level to prevent data leakage and get trustable results. This assures that the training and validation sets to be completely independent and confirms that no information is leaking from the test set into the training set during the development of the model. Additionally, employing 3D models for 3D data with subject-level train-test split should be encouraged as 2D models do not effectively capture 3D features. The high computational complexity of 3D models may be tackled using image patches or sub-images, and parallel processing on multiple GPUs, or, in some cases, by image downsampling.

With recent advances in machine learning, more and more people are becoming interested in applying these techniques to biomedical imaging, and there is a real and growing risk that not all researchers pay sufficient attention to this serious issue. We also emphasize the need to document how the CV is implemented, the architecture used, how the different hyperparameter choices/tunings are made and include their values where possible. Besides, we advocate reproducibility and encourage the community to take a step towards transparency in deep/machine learning in medical image analysis by publicly releasing code, including containers and a link to open datasets ${ }^{59}$. Moreover, a blind evaluation on external test sets-i.e., within open challenges-is highly recommended.

One limitation of this study is due to the substantial overfitting we observed while applying a subject-level split for training our models. This overfitting is manifested by the very high accuracy in training sets compared to that observed in test sets (Table 4). Focussing our efforts on alleviating overfitting may have improved performance in the test set, thus reducing the extent of the faulty boost due to the slice-level split. Moreover, in this study, we have not assessed all data leakage types, including late split and hyperparameters optimization in the test set-that may also be present in 3D CNN studies. We have found evidence of all these data leakage issues in the recent literature (see Tables 1,2,3), and we plan to quantify their effect in our future work systematically.

In conclusion, training a $2 \mathrm{D} \mathrm{CNN}$ model for analyzing $3 \mathrm{D}$ brain image data must be performed using a subject-level CV to prevent data leakage. The adoption of slice-based CV results in very optimistic model performances, especially for small datasets, as the extent of the overestimation due to data leakage is severe.

\section{Materials and methods}

Datasets. In this study, we adopted the scans collected by three public and international datasets of $\mathrm{T}_{1}$-weighted images of patients with AD (the OASIS dataset ${ }^{53}$ and the ADNI dataset ${ }^{54}$ ) and de-novo PD (the PPMI datase ${ }^{55}$ ). An additional private de-novo PD dataset, namely the Versilia dataset, has also been used. A summary of the demographics of the datasets used in this study is shown in Table 5. In the following sections, a detailed description of all datasets will be reported.

OASIS-200, OASIS-34, and OASIS-random datasets. We have used the $\mathrm{T}_{1}$-weighted images of $100 \mathrm{AD}$ patients [(59 women and 41 men, age $76.70 \pm 7.10$ years, mean \pm standard deviation (SD)] and 100 healthy controls (73 women and 27 men, age $75.50 \pm 9.10$ years, mean \pm SD) from the OASIS- 1 study-a cross-sectional cohort of the OASIS brain MRI dataset ${ }^{53}$, freely available at https://www.oasis-brains.org/. In particular, we have employed the same scans that were previously selected by other authors ${ }^{21}$. We called this dataset OASIS-200. The subject identification numbers (IDs) and demographics of these subjects were specified in Supplementary Table S6. No significant difference in age ( $\mathrm{p}=0.15$ at $\mathrm{t}$-test) was found between the two groups, while a significant (borderline) difference in gender was observed ( $\mathrm{p}=0.04$ at $\chi^{2}$-test).

In OASIS-1, AD diagnosis, as well as the severity of the disease, were evaluated based on the global Clinical Dementia Rating (CDR) score derived from individual CDR scores for the domains memory, orientation, judgment and problem solving, function in community affairs, home and hobbies, and personal case ${ }^{60,61}$. Subjects with a global CDR score of 0 have been labeled as healthy controls, while scores 0.5 (very mild), 1 (mild), 2 (moderate), and 3 (severe) have been all labeled as AD.

All $\mathrm{T}_{1}$-weighted images have been acquired on a 1.5 T MR scanner (Vision, Siemens, Erlangen, Germany), using a Magnetization Prepared Rapid Gradient Echo (MPRAGE) sequence in a sagittal plane [repetition time $(\mathrm{TR})=9.7 \mathrm{~ms}$, echo time $(\mathrm{TE})=4.0 \mathrm{~ms}$, flip angle $=10^{\circ}$, inversion time $(\mathrm{TI})=20 \mathrm{~ms}$, delay time $(\mathrm{TD})=200 \mathrm{~ms}$, voxel size $=1 \mathrm{~mm} \times 1 \mathrm{~mm} \times 1.25 \mathrm{~mm}$, matrix size $=256 \times 256$, number of slices $=128]^{53}$.

ADNI dataset. We considered the $\mathrm{T}_{1}$-weighted MRI data of $100 \mathrm{AD}$ patients (44 women and 56 men, age $74.28 \pm 7.96$ years, mean \pm SD) and 100 healthy controls (52 women and 48 men, age $75.04 \pm 7.11$ years, mean $\pm S D)$. No significant difference in age $(p=0.24$ at $t$-test $)$ and gender $\left(p=0.26\right.$ at $\chi^{2}$-test $)$ was found between the two groups. Alzheimer's disease patients have been randomly chosen from the ADNI 2 dataset (available at http://adni.loni.usc.edu/) - acohort of ADNI that extends the work of ADNI 1 and ADNI-GO studies ${ }^{54}$. Led by 


\begin{tabular}{|c|c|c|}
\hline Dataset & Patients & Healthy controls \\
\hline \multicolumn{3}{|l|}{ OASIS-200 } \\
\hline Number of subjects & 100 & 100 \\
\hline Age (range, years) & $62-96$ & $59-94$ \\
\hline Age (mean $\pm S D$, years) & $76.70 \pm 7.10$ & $75.50 \pm 9.10$ \\
\hline Gender (women/men) & $59 / 41$ & $73 / 27$ \\
\hline \multicolumn{3}{|l|}{ ADNI } \\
\hline Number of subjects & 100 & 100 \\
\hline Age (range, years) & $56-89$ & $58-95$ \\
\hline Age (mean $\pm S D$, years) & $74.28 \pm 7.96$ & $75.04 \pm 7.11$ \\
\hline Gender (women/men) & $44 / 56$ & $52 / 48$ \\
\hline \multicolumn{3}{|l|}{ PPMI } \\
\hline Number of subjects & 100 & 100 \\
\hline Age (range, years) & $34-82$ & $31-83$ \\
\hline Age (mean $\pm S D$, years) & $61.71 \pm 9.99$ & $61.91 \pm 11.52$ \\
\hline Gender (women/men) & $40 / 60$ & $36 / 64$ \\
\hline \multicolumn{3}{|l|}{ Versilia } \\
\hline Number of subjects & 17 & 17 \\
\hline Age (range, years) & $48-78$ & $54-77$ \\
\hline Age (mean $\pm S D$, years) & $64 \pm 7.21$ & $64.00 \pm 7.00$ \\
\hline Gender (women/men) & $4 / 13$ & $5 / 12$ \\
\hline
\end{tabular}

Table 5. Demographic features of subjects belonging to OASIS-200, ADNI, PPMI, and Versilia datasets. The same information for the OASIS-34 datasets has been reported in Supplementary Table S5. AD Alzheimer's disease, $A D N I$ Alzheimer's Disease Neuroimaging Initiative, OASIS open access series of imaging studies, $P D$ Parkinson's disease, PPMI Parkinson's Progression Markers Initiative, SD standard deviation.

Principal Investigator Michael W. Weiner, MD, ADNI was launched in 2003 to investigate if biological markers (such as MRI and PET) can be combined to define the progression of MCI and early AD. We have used MPRAGE $\mathrm{T}_{1}$-weighted MRI scans acquired by $3 \mathrm{~T}$ scanners [6 Siemens (Erlangen, Germany) MRI scanners and 6 Philips (Amsterdam, Netherlands) scanners] in a sagittal plane (voxel size $=1 \mathrm{~mm} \times 1 \mathrm{~mm} \times 1.2 \mathrm{~mm}$ ). The image size of the $\mathrm{T}_{1}$-weighted data acquired from the Siemens and Philips scanners were $176 \times 240 \times 256$ and $170 \times 256 \times 256$, respectively. Since ADNI 2 is a longitudinal dataset, more than one scan was available for each subject. The first scan of each participant has been chosen to produce a cross-sectional dataset. Supplementary Table S7 provides subject IDs and the acquisition date of the specific scan used in our study. The MRI acquisition protocol for each MRI scanner can be found at http://adni.loni.usc.edu/methods/documents/mri-protocols/. In ADNI 2 dataset, subjects have been categorized as AD patients or healthy controls based on whether subjects have complaints about their memory and by considering a combination of neuropsychological clinical scores ${ }^{54}$.

PPMI dataset. We randomly selected 100 de-novo PD subjects (40 women and 60 men, age $61.71 \pm 9.99$, mean \pm SD) and 100 healthy controls (36 women and 64 men, age $61.91 \pm 11.52$, mean \pm SD) from the publicly available PPMI dataset (https://ida.loni.usc.edu/login.jsp?project=PPMI). No significant difference in age $(p=0.44$ at $t$-test $)$ and gender $\left(p=0.56\right.$ at $\chi^{2}$-test $)$ was found between the two groups. The criterion used to recruit de-novo PD patients, and healthy controls were defined by Marek et al..$^{55}$. Briefly, PD patients were selected within two years of diagnosis with a Hoehn and Yahr score $<3^{62}$, at least two of resting tremor, either bradykinesia or rigidity (must have either resting tremor or asymmetric bradykinesia) or a single asymmetric resting tremor or asymmetric bradykinesia and dopamine transporter (DAT) or vesicular monoamine transporter type 2 (VMAT-2) imaging showing a dopaminergic deficit. Healthy controls were free from any clinically significant neurological disorder ${ }^{55}$.

The $\mathrm{T}_{1}$-weighted scans were collected at baseline using MR scanners manufactured by Siemens (11 scanners at $3 \mathrm{~T}$ and five scanners at $1.5 \mathrm{~T}$ ), Philips Medical Systems (10 scanners at $3 \mathrm{~T}$ and 11 scanners at $1.5 \mathrm{~T})$, GE Medical Systems ( 11 scanners at $3 \mathrm{~T}$ and 24 scanners at $1.5 \mathrm{~T}$ ) and another anonymous one ( 5 scanners at $1.5 \mathrm{~T}$ ). We also found three subjects whose MRI protocol was missing. The details of the MRI protocols of all scanners can be found in Supplementary Table S8.

Versilia dataset. Seventeen ( 4 women and 13 men, age $64 \pm 7.21$ years, mean \pm SD) patients with de-novo parkinsonian syndrome consecutively referred to a Neurology Unit to evaluate PD over a 24-month interval (from June 2012 to June 2014) were recruited in this dataset. More details about clinical evaluation can be found in Ref. ${ }^{63}$. Seventeen healthy controls ( 5 women and 12 men, age $64 \pm 7$ years, mean \pm SD) with no history of neurological diseases and normal neurological examination were recruited as controls. No significant difference in age $(\mathrm{p}=0.95$ at $\mathrm{t}$-test $)$ and gender $\left(\mathrm{p}=0.70\right.$ at $\chi^{2}$-test $)$ was found between the two groups

All subjects underwent high-resolution 3D $\mathrm{T}_{1}$-weighted imaging on a 1.5 T MR scanner system (Magnetom Avanto, software version Syngo MR B17, Siemens, Erlangen-Germany) equipped with a 12-element matrix 


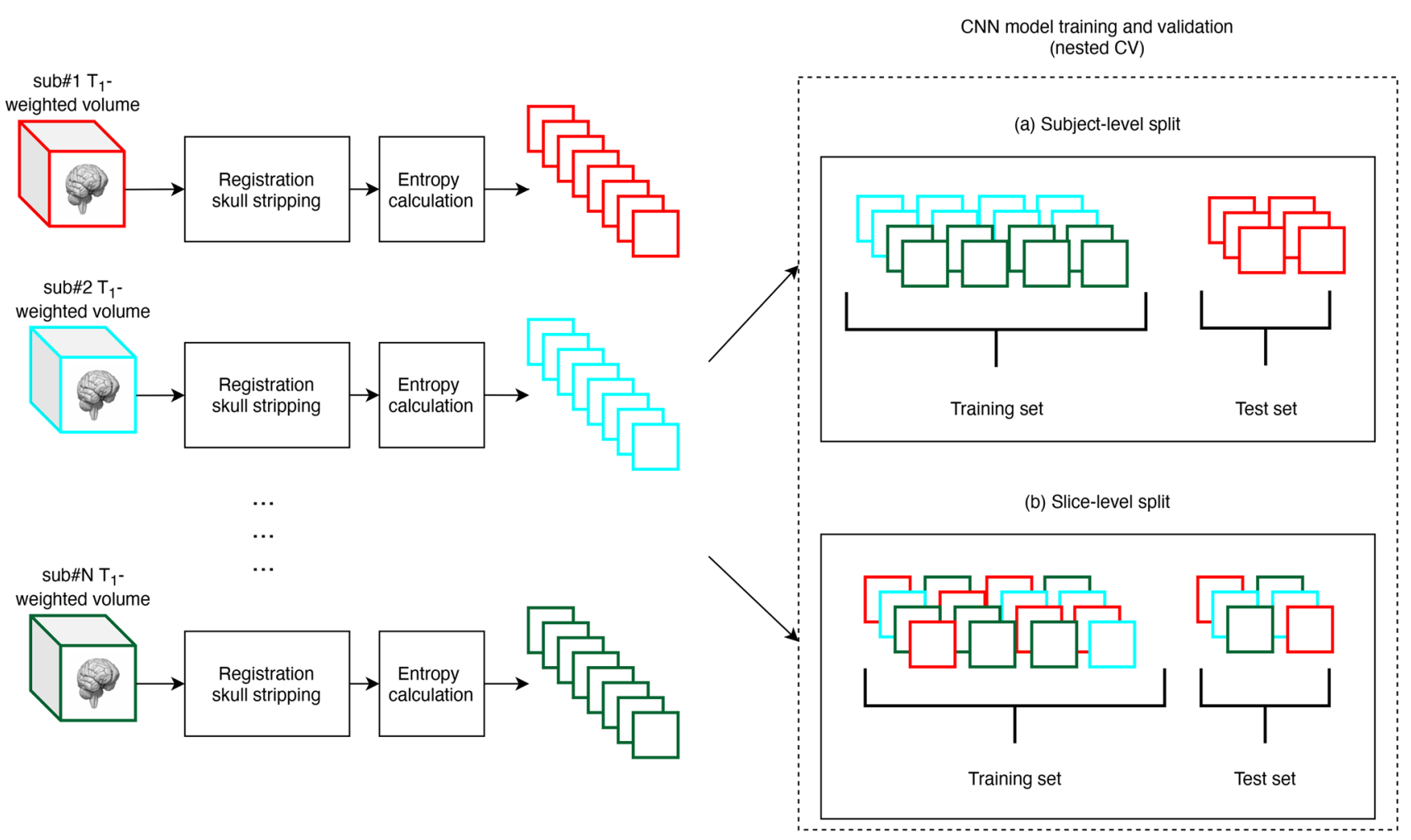

Figure 1. Schematic diagram of the overall $\mathrm{T}_{1}$-weighted MRI data processing and validation scheme. First, a preprocessing stage included co-registration to a standard space, skull-stripping and slices selection based on entropy calculation. Then, CNNs model's training and validation have been performed on each dataset in a nested CV loop using two different data split strategies: (a) subject-level split, in which all the slices of a subject have been placed either in the training or in the test set, avoiding any form of data leakage; (b) slice-level split, in which all the slices have been pooled together before $\mathrm{CV}$, then split randomly into training and test set.

radiofrequency head coil and SQ-engine gradients. The SQ-engine gradients had a maximum strength of 45 $\mathrm{mT} / \mathrm{m}$ and a slew rate of $200 \mathrm{~T} / \mathrm{m} / \mathrm{s}$. $\mathrm{T}_{1}$-weighted $\mathrm{MR}$ images were acquired with an axial high resolution 3D MPRAGE sequence with $\mathrm{TR}=1900 \mathrm{~ms}, \mathrm{TE}=3.44 \mathrm{~ms}, \mathrm{TI}=1100 \mathrm{~ms}$, flip angle $=15^{\circ}$, slice thickness $=0.86 \mathrm{~mm}$, field of view $(\mathrm{FOV})=220 \mathrm{~mm} \times 220 \mathrm{~mm}$, matrix size $=256 \times 256$, number of excitations $(\mathrm{NEX})=2$, number of slices $=176$.

$\mathrm{T}_{1}$-weighted MRI data preprocessing. All $\mathrm{T}_{1}$-weighted MRI data went through two preprocessing steps (see Fig. 1). In the first stage, co-registration to a standard template space and skull stripping were applied to re-align all the images and remove non-brain regions. In the second stage, a subset of axial images has been collected using an entropy-based slice selection approach.

Co-registration to a standard template space and skull stripping. For the OASIS datasets, we used publicly available preprocessed data (gain-field corrected, brain masked, and co-registration) ${ }^{64}$. Briefly, the brain masks from OASIS were obtained using an atlas-registration-based method, and their quality was controlled by human experts $^{53}$, and each volume has been co-registered to the Talairach and Tournoux atlas. Each preprocessed $\mathrm{T}_{1}$-weighted volume had a data matrix size of $176 \times 208 \times 176$ and a voxel size of $1 \mathrm{~mm} \times 1 \mathrm{~mm} \times 1 \mathrm{~mm}^{64}$.

For all other datasets, we have co-registered each individual $\mathrm{T}_{1}$-weighted volume to the MNI152 standard template space (at $1 \mathrm{~mm}$ voxel size-available in the FSL version 6.0.3 package) by using the SyN algorithm included in ANTs package (version 2.1.0) with default parameters ${ }^{65}$. Then, the brain mask of the standard template space has been applied to each co-registered volume. Each preprocessed $\mathrm{T}_{1}$-weighted volume had a data matrix size of $182 \times 218 \times 182$ and a voxel size of $1 \mathrm{~mm} \times 1 \mathrm{~mm} \times 1 \mathrm{~mm}$.

Supplementary Figure $\mathrm{S} 1$ online illustrates sample preprocessed $\mathrm{T}_{1}$-weighted slices from OASIS-200, ADNI, PPMI, and Versilia datasets.

Entropy-based slice selection. Each $\mathrm{T}_{1}$-weighted slice generally conveys a different amount of information. Given that we are interested in developing a 2D CNN model, we have performed a preliminary slice selection based on the amount of information. More specifically, for each $\mathrm{T}_{1}$-weighted volume, the Shannon entropy $E_{S}$, representing the information content, was computed for each axial slice, as follows: 

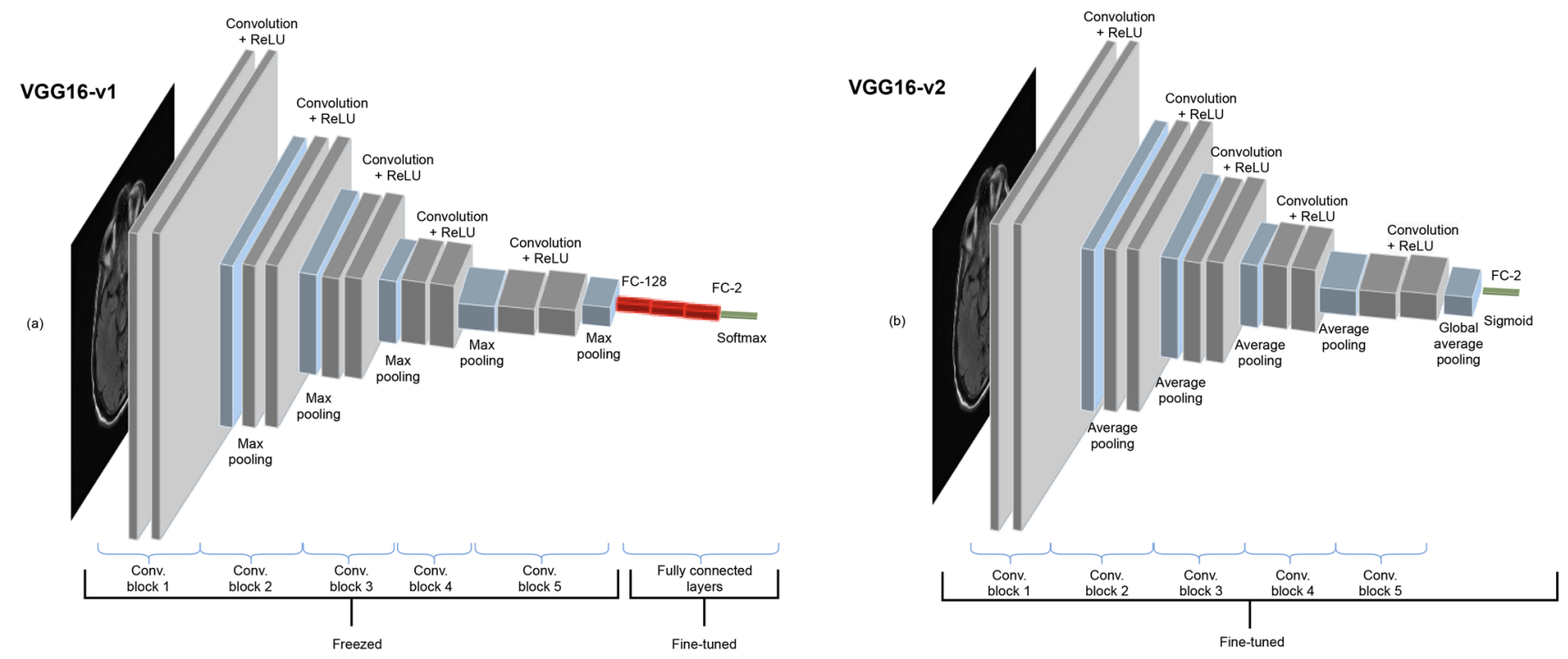

Figure 2. The two different networks based on the VGG16 architecture are shown. Each colored block of layers illustrates a series of convolutions. (a) The first model, named as VGG16-v1 consists of five convolutional blocks followed by three fully connected layers. Only the last three fully connected layers are fine-tuned. (b) On the other hand, the second model, VGG16-v2, has five convolutional blocks followed by a global average pooling layer, and all the layers are fine-tuned.

$$
E_{S}=\sum_{k} p_{k} \log _{2}\left(p_{k}\right)
$$

where $k$ is the number of grayscale levels in the slice and $p_{k}$ is the probability of occurrence, estimated as the relative frequency in the image, for the gray level $k$. Then, for each $\mathrm{T}_{1}$-weighted volume, the slices were ordered in descending order based on their entropy scores, and, finally, we selected only the eight axial slices that showed the highest entropy ${ }^{21}$.

To be consistent with the input sizes of the proposed 2D CNN models, all slices were resized to $224 \times 224$ pixels by fitting a cubic spline between the 4 -by- 4 neighborhood pixels ${ }^{66}$. Voxel-wise feature standardization has also been applied to make training the CNNs easier and achieve faster convergence, i.e., for each voxel, an average value of all grayscale values within the brain mask has been subtracted and scaled by the standard deviation (within the brain mask).

Model architectures. Since the number of subjects of each dataset may not be sufficient to train with high accuracy a 2D CNN model from scratch, we have used a machine learning technique called transfer learning that allows employing pre-trained models, i.e., model parameters previously developed for one task (source domain) to be transferred to target domain for weight initialization and feature extraction. In particular, CNN layers hierarchically extract features starting from the general low-level features to those specific to the target class, and, using transfer learning, the general low-level features can be shared across tasks. Notably, we used pre-trained VGG16 $6^{67}$ and ResNet- $18^{68}$ models in this study, as detailed in the following sections. The transfer learning approach and VGG16 architectures used in this study are similar to those employed in Ref. ${ }^{21}$ as their results triggered our investigation of data leakage.

VGG16-based models. VGG16 is one of the most influential architectures which explores network depth with very small $(3 \times 3)$ convolution filters stacked on top of each other. VGG16 consists of five convolutional blocks, with alternating convolutional and pooling layers and three fully-connected layers.

In transfer learning, the most common approach is copying the first $n$ layers of the pre-trained network to the first $n$ layers of a target network and then randomly initializing the remaining layers to be trained on the target task. Depending on the size of the target dataset and the number of parameters in the first $n$ layers, these copied features can be left unchanged (i.e., frozen) or fine-tuned during the training of the network on a new dataset. It is well accepted that if the target dataset is relatively small, fine-tuning may cause overfitting, whereas if the target dataset is large, then the base features can be fine-tuned to improve the model's performance without overfitting.

To investigate the effect of fine-tuning, we have tested two different variants of VGG16 architecture, namely VGG16-v1 and VGG16-v2 (Fig. 2). The former model has been used as a feature extractor where the weights for all network layers are frozen except that of the final fully connected layer. Randomly initialized fully connected layers have replaced the three topmost layers with rectified linear unit (ReLU) activation. The weights are initialized according to the Xavier initialization heuristic ${ }^{69}$ to prevent the gradients from vanishing or exploding.

The VGG16-v2 model has been utilized as a weight initializer where the weights are derived from the pretrained network and fine-tuned during training. We have replaced the fully connected layers with a randomly 

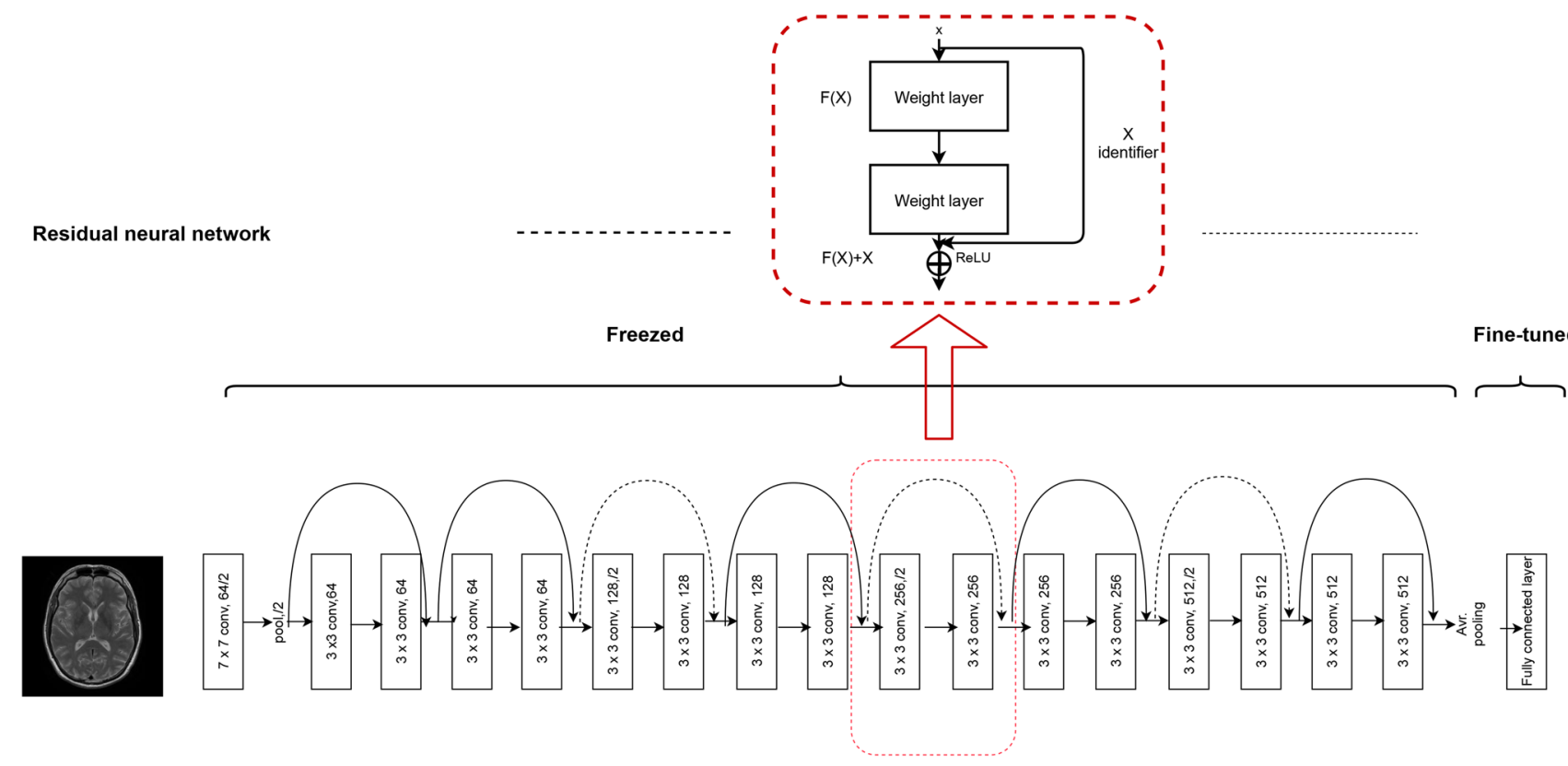

Figure 3. A modified ResNet-18 architecture with an average pooling layer at the end is shown. The upper box represents a residual learning block with an identity shortcut. Each layer is denoted as (filter size, \# channels); layers labeled as "freezed" indicates that the weights are not updated during backpropagation, whereas when they are labeled as "fine-tuned" they are updated. The identity shortcuts can be directly used when the input and output are of the same dimensions (solid line shortcuts) and when the dimensions increase (dotted line shortcuts). ReLU rectified linear unit.

initialized global average pooling (GAP) layer suggested by Lin et al. ${ }^{70}$ to reduce the number of parameters and, rather than freezing the $\mathrm{CNN}$ layers, we have fine-tuned all layers.

ResNet-18 based model. It has been long believed that deeper networks can learn more complex nonlinear relationships than shallower networks with the same number of neurons, and thus network depth is of great importance on model performance ${ }^{71}$. However, many studies revealed that deeper networks often converge at a higher training and test error rate when compared to their shallower counterparts ${ }^{68}$. Therefore, stacking more layers to the plain networks may eventually degrade the model's performance while complicating the optimization process. To overcome this issue, He and colleagues introduced deep residual neural networks and achieved top-5 test accuracies with their models on the popular ImageNet test set ${ }^{68}$. The model was proposed as an attempt to solve the vanishing gradients and the degradation problems using residual blocks. With these residual blocks, the feature of any deeper unit can be computed as the sum of the activation of a shallower unit and the residual function. This architecture causes the gradient to be directly propagated to shallower units making ResNets easier to train.

There are different versions of ResNet architecture with various numbers of layers. In this work, we used ResNet-18 architecture, an 18-layer residual deep learning network consisting of five stages, each with a convolution and identity block ${ }^{68}$. In our model, one fully connected layer with sigmoid activation has been added at the end of the network- a common practice in binary classification tasks as it takes a real-valued input and squashes the output to a range between 0 and 1 . Since the network is relatively smaller and has a lower number of parameters than VGG16, the weights and biases of all the transferred layers are fine-tuned while the newly added fully connected layer has been trained to start from randomly initialized weights. The architecture of our ResNet-18 model can be seen in Fig. 3.

Model training and validation. Each $2 \mathrm{D} \mathrm{CNN}$ model has been trained and validated using a nested CV strategy - a validation scheme that allows examining the unbiased generalization performance of the trained models along with performing, at the same time, hyperparameters optimization ${ }^{29}$. It involves nesting two $k$-fold $\mathrm{CV}$ loops where the inner loop is used for optimizing model hyperparameters, and the outer loop gives an unbiased estimate of the performance of the best model. It is especially suitable when the amount of data available is insufficient to allow separate validation and test $\operatorname{sets}^{29}$. A schematic diagram of the procedure is illustrated in Supplementary Fig. S2. It starts by dividing the dataset into $k$ folds, and onefold is kept as a test set (outer CV), while the other $k-1$ folds are split into inner folds (inner CV). The model hyperparameters are chosen from the hyperparameter space through a grid search based on the average performance of the model over the inner folds. In particular, we varied the learning rate in the set $\left\{10^{-5}, 3 \times 10^{-5}, 10^{-4}, 3 \times 10^{-4}, 10^{-3}\right\}$ and the learning rate decay in $\{0,0.1,0.3,0.5\}$. The chosen model is then fitted with all the outer fold training data and tested on the unseen test fold, resulting in an unbiased estimation of the model's prediction error. Specifically, we choose a tenfold CV because it offers a favorable bias-variance tradeoff ${ }^{72,73}$. 
In all experiments, we used batch size $=128$ and epoch number $=50$. Due to its ability to adaptively updating individual learning rates for each parameter, an Adam optimizer was used ${ }^{74}$. Each selected slice of the 3D $\mathrm{T}_{1}$-weighted volume has been classified independently and the final model's performance was stated using the mean slice-level accuracy, separately, on the training set and test set folds of the outer CV.

We thus conducted CNNs model's training and validation on each dataset in a nested CV loop using two different data split strategies: (a) subject-level split, in which all the slices of a subject have been placed either in the training set or in the test set, avoiding any form of data leakage; (b) slice-level split, in which all the slices have been pooled together before $\mathrm{CV}$, then split randomly into training and test set. In this case, for each slice of the test set, a set of highly correlated slices coming from the MR volume of the same subject ended up in the training set, giving rise to data leakage, as shown pictographically in Fig. 1.

CNN models were carried out using a custom-made software in Python language (version 3.6.8) using the following modules: CUDA v.9.0.176 ${ }^{75}$, TensorFlow-gpu v.1.12.0 ${ }^{76}$, Keras v.2.2.4 ${ }^{77}$, Scikit-learn v.0.20.2 ${ }^{78}$, Nibabel v.2.3.3 $3^{79}$, and OpenCV v.3.3.066. All the source code can be found in a Github repository at https://github.com/ Imaging-AI-for-Health-virtual-lab/Slice-Level-Data-Leakage, and a Docker image can be downloaded at https:// hub.docker.com/repository/docker/ai4healthvlab/slice-level-data-leakage. The training and validation of CNN models were performed on a workstation equipped with a 12 GB G5X frame buffer NVIDIA TITAN X (Pascal) GPU with 64 GB RAM, 8 CPUs, 3584 CUDA cores and 11.4 Gbps processing speed. The average computational time for CNN training on a dataset of 34 and 200 subjects were $5.68 \mathrm{~h}$ (VGG16-v1), $5.63 \mathrm{~h}$ (VGG16-v2), $2.94 \mathrm{~h}$ (ResNet-18) and $33.93 \mathrm{~h}$ (VGG16-v1), $33.82 \mathrm{~h}$ (VGG16-v2), $14.12 \mathrm{~h}$ (ResNet-18), respectively. The total computational time for this study was thus about 17 days.

Received: 26 April 2021; Accepted: 28 October 2021

Published online: 19 November 2021

\section{References}

1. Hatcher, W. G. \& Yu, W. A survey of deep learning: Platforms, applications and emerging research trends. IEEE Access 6, 2441124432 (2018).

2. Goodfellow, I., Bengio, Y. \& Courville, A. Deep Learning (The MIT Press, 2016)

3. Greenspan, H., van Ginneken, B. \& Summers, R. M. Guest editorial deep learning in medical imaging: Overview and future promise of an exciting new technique. IEEE Trans. Med. Imaging 35, 1153-1159 (2016).

4. Zaharchuk, G., Gong, E., Wintermark, M., Rubin, D. \& Langlotz, C. P. Deep learning in neuroradiology. Am. J. Neuroradiol. 39, 1776-1784 (2018).

5. Bahrami, K. et al. Reconstruction of 7T-like images from 3T MRI. IEEE Trans. Med. Imaging 35, 2085-2097 (2016).

6. Han, X. MR-based synthetic CT generation using a deep convolutional neural network method. Med. Phys. 44, 1408-1419 (2017).

7. Li, R. et al. Deep learning based imaging data completion for improved brain disease diagnosis. In MICCAI International Conference on Medical Image Computing and Computer-Assisted Intervention, Vol. 17, 305-312 (2014).

8. Liu, F., Jang, H., Kijowski, R., Bradshaw, T. \& McMillan, A. B. Deep learning MR imaging-based attenuation correction for PET/ MR imaging. Radiology 286, 676-684 (2018).

9. Vemulapalli, R. Deep Networks and Mutual Information maximization for Cross-modal Medical Image Synthesis 381-403 (Elsevier, 2017).

10. Zhu, B., Liu, J. Z., Cauley, S. F., Rosen, B. R. \& Rosen, M. S. Image reconstruction by domain-transform manifold learning. Nature 555, 487-492 (2018).

11. Chang, P. D. Fully convolutional deep residual neural networks for brain tumor segmentation. In Brainlesion: Glioma, Multiple Sclerosis, Stroke and Traumatic Brain Injuries Vol. 10154 (eds Crimi, A. et al.) 108-118 (Springer, 2016).

12. Dou, Q. et al. Automatic detection of cerebral microbleeds from MR images via 3D convolutional neural networks. IEEE Trans. Med. Imaging 35, 1182-1195 (2016).

13. Maier, O., Schröder, C., Forkert, N. D., Martinetz, T. \& Handels, H. Classifiers for ischemic stroke lesion segmentation: A comparison study. PLoS ONE 10, e0145118 (2015).

14. Liu, S. et al. Multimodal neuroimaging feature learning for multiclass diagnosis of Alzheimer's Disease. IEEE Trans. Biomed. Eng. 62, 1132-1140 (2015).

15. Plis, S. M. et al. Deep learning for neuroimaging: A validation study. Front. Neurosci. https://doi.org/10.3389/fnins.2014.00229 (2014).

16. Davatzikos, C. Machine learning in neuroimaging: Progress and challenges. Neuroimage 197, 652-656 (2019).

17. Liu, S. et al. Early diagnosis of Alzheimer's disease with deep learning. In 2014 IEEE 11th International Symposium on Biomedical Imaging (ISBI), 1015-1018. https://doi.org/10.1109/ISBI.2014.6868045 (IEEE, 2014).

18. Suk, H.-I. \& Shen, D. Deep learning-based feature representation for AD/MCI classification. In MICCAI International Conference on Medical Image Computing and Computer-Assisted Intervention, Vol. 16, 583-590 (2013).

19. Kuang, D., Guo, X., An, X., Zhao, Y. \& He, L. Discrimination of ADHD based on fMRI data with deep belief network. In Intelligent Computing in Bioinformatics (eds Huang, D.-S. et al.) 225-232 (Springer, 2014).

20. Vieira, S., Pinaya, W. H. L. \& Mechelli, A. Using deep learning to investigate the neuroimaging correlates of psychiatric and neurological disorders: Methods and applications. Neurosci. Biobehav. Rev. 74, 58-75 (2017).

21. Hon, M. \& Khan, N. Towards Alzheimer's disease classification through transfer learning. http://arXiv.org/1711.11117 (2017).

22. Sarraf, S., DeSouza, D. D., Anderson, J. \& Tofighi, G. DeepAD: Alzheimer's disease classification via deep convolutional neural networks using MRI and fMRI. BioRxiv. https://doi.org/10.1101/070441 (2017).

23. Wu, C. et al. Discrimination and conversion prediction of mild cognitive impairment using convolutional neural networks. Quant. Imaging Med. Surg. 8, 992-1003 (2018).

24. Islam, J. \& Zhang, Y. Brain MRI analysis for Alzheimer's disease diagnosis using an ensemble system of deep convolutional neural networks. Brain Inform. 5, 2 (2018).

25. Esmaeilzadeh, S., Yang, Y. \& Adeli, E. End-to-end Parkinson disease diagnosis using brain MR-images by 3D-CNN. http://arXiv. org/1806.05233 (2018).

26. Sivaranjini, S. \& Sujatha, C. M. Deep learning based diagnosis of Parkinson's disease using convolutional neural network. Multimedia Tools Appl. https://doi.org/10.1007/s11042-019-7469-8 (2019).

27. Kaufman, S., Rosset, S. \& Perlich, C. Leakage in data mining: Formulation, detection, and avoidance. In Proc. 17th ACM SIGKDD International Conference on Knowledge Discovery and Data Mining_KDD'11, 556. https://doi.org/10.1145/2020408.2020496 (ACM Press, 2011). 
28. Reunanen, J. Overfitting in making comparisons between variable selection methods. J. Mach. Learn. Res 3, 1371-1382 (2003). 29. Varma, S. \& Simon, R. Bias in error estimation when using cross-validation for model selection. BMC Bioinform. 7, 91 (2006).

30. Wen, J. et al. Convolutional neural networks for classification of Alzheimer's disease: Overview and reproducible evaluation. Med. Image Anal. 63, 101694 (2020).

31. Winkler, J. K. et al. Association between surgical skin markings in dermoscopic images and diagnostic performance of a deep learning convolutional neural network for melanoma recognition. JAMA Dermatol. 155, 1135 (2019).

32. Oakden-Rayner, L., Dunnmon, J., Carneiro, G. \& Re, C. Hidden stratification causes clinically meaningful failures in machine learning for medical imaging. In Proc. ACM Conference on Health, Inference, and Learning, 151-159. https://doi.org/10.1145/33685 55.3384468 (ACM, 2020).

33. Narla, A., Kuprel, B., Sarin, K., Novoa, R. \& Ko, J. Automated classification of skin lesions: From pixels to practice. J. Investig. Dermatol. 138, 2108-2110 (2018).

34. Blum, A., Kalai, A. \& Langford, J. Beating the hold-out: Bounds for K-fold and progressive cross-validation. In Proc. Twelfth Annual Conference on Computational Learning Theory-COLT'99, 203-208. https://doi.org/10.1145/307400.307439 (ACM Press, 1999).

35. Yadav, S. \& Shukla, S. Analysis of k-fold cross-validation over hold-out validation on colossal datasets for quality classification. In 2016 IEEE 6th International Conference on Advanced Computing (IACC), 78-83. https://doi.org/10.1109/IACC.2016.25 (IEEE, 2016).

36. Gunawardena, K. A. N. N. P., Rajapakse, R. N. \& Kodikara, N. D. Applying convolutional neural networks for pre-detection of Alzheimer's disease from structural MRI data. In 2017 24th International Conference on Mechatronics and Machine Vision in Practice (M2VIP), 1-7. https://doi.org/10.1109/M2VIP.2017.8211486 (2017).

37. Jain, R., Jain, N., Aggarwal, A. \& Hemanth, D. J. Convolutional neural network based Alzheimer's disease classification from magnetic resonance brain images. Cogn. Syst. Res. 57, 147 (2019).

38. Khagi, B., Lee, B., Pyun, J.-Y. \& Kwon, G.-R. CNN models performance analysis on MRI images of OASIS dataset for distinction between Healthy and Alzheimer's patient. In 2019 International Conference on Electronics, Information, and Communication (ICEIC), 1-4. https://doi.org/10.23919/ELINFOCOM.2019.8706339 (IEEE, 2019).

39. Wang, S., Shen, Y., Chen, W., Xiao, T.-F. \& Hu, J. Automatic recognition of mild cognitive impairment from MRI images using expedited convolutional neural networks. In ICANN. https://doi.org/10.1007/978-3-319-68600-4_43 (2017).

40. Puranik, M., Shah, H., Shah, K. \& Bagul, S. Intelligent Alzheimer's detector using deep learning. In 2018 Second International Conference on Intelligent Computing and Control Systems (ICICCS), 318-323. https://doi.org/10.1109/ICCONS.2018.8663065 (IEEE, 2018).

41. Basheera, S. \& Sai Ram, M. S. Convolution neural network-based Alzheimer's disease classification using hybrid enhanced independent component analysis based segmented gray matter of T2 weighted magnetic resonance imaging with clinical valuation. Alzheimer's Dementia 5, 974-986 (2019).

42. Nawaz, A. et al. Deep convolutional neural network based classification of Alzheimer's disease using MRI data. In 2020 IEEE 23rd International Multitopic Conference (INMIC), 1-6. https://doi.org/10.1109/INMIC50486.2020.9318172 (IEEE, 2020).

43. Farooq, A., Anwar, S., Awais, M. \& Rehman, S. A deep CNN based multi-class classification of Alzheimer's disease using MRI. In 2017 IEEE International Conference on Imaging Systems and Techniques (IST), 1-6. https://doi.org/10.1109/IST.2017.8261460 (2017).

44. Ramzan, F. et al. A deep learning approach for automated diagnosis and multi-class classification of Alzheimer's disease stages using resting-state fMRI and residual neural Networks. J. Med. Syst. 44, 37 (2019).

45. Raza, M. et al. Diagnosis and monitoring of Alzheimer's patients using classical and deep learning techniques. Expert Syst. Appl. 136, 353-364 (2019).

46. Pathak, K. C. \& Kundaram, S. S. Accuracy-based performance analysis of Alzheimer's disease classification using deep convolution neural network. In Soft Computing: Theories and Applications Vol. 1154 (eds Pant, M. et al.) 731-744 (Springer, 2020).

47. Libero, L. E., DeRamus, T. P., Lahti, A. C., Deshpande, G. \& Kana, R. K. Multimodal neuroimaging based classification of autism spectrum disorder using anatomical, neurochemical, and white matter correlates. Cortex 66, 46-59 (2015).

48. Zhou, Y., Yu, F. \& Duong, T. Multiparametric MRI characterization and prediction in autism spectrum disorder using graph theory and machine learning. PLoS ONE 9, e90405 (2014).

49. Lui, Y. W. et al. Classification algorithms using multiple MRI features in mild traumatic brain injury. Neurology 83, 1235-1240 (2014).

50. Hasan, A. M., Jalab, H. A., Meziane, F., Kahtan, H. \& Al-Ahmad, A. S. Combining deep and handcrafted image features for MRI brain scan classification. IEEE Access 7, 79959-79967 (2019).

51. Al-Khuzaie, F. E. K., Bayat, O. \& Duru, A. D. Diagnosis of Alzheimer disease using 2D MRI slices by convolutional neural network. Appl. Bionics Biomech. 2021, 6690539 (2021).

52. Yagis, E., De Herrera, A. G. S. \& Citi, L. Generalization performance of deep learning models in neurodegenerative disease classification. In 2019 IEEE International Conference on Bioinformatics and Biomedicine (BIBM), 1692-1698. https://doi.org/10.1109/ BIBM47256.2019.8983088 (IEEE, 2019).

53. Marcus, D. S. et al. Open access series of imaging studies (OASIS): Cross-sectional MRI data in young, middle aged, nondemented, and demented older adults. J. Cogn. Neurosci. 19, 1498-1507 (2007).

54. Petersen, R. C. et al. Alzheimer's Disease Neuroimaging Initiative (ADNI). Neurology 74, 201-209 (2010).

55. Marek, K. et al. The Parkinson's progression markers initiative (PPMI)-Establishing a PD biomarker cohort. Ann. Clin. Transl. Neurol. 5, 1460-1477 (2018).

56. Murad, M. et al. Efficient reconstruction technique for multi-slice CS-MRI using novel interpolation and 2D sampling scheme. IEEE Access 8, 117452-117466 (2020).

57. Suk, H.-I., Shen, D. \& Alzheimer's Disease Neuroimaging Initiative Deep learning in diagnosis of brain disorders. In Recent Progress in Brain and Cognitive Engineering Vol. 5 (eds Lee, S.-W. et al.) 203-213 (Springer, 2015).

58. Kobayashi, S., Kane, T. \& Paton, C. The privacy and security implications of open data in Healthcare: A contribution from the IMIA open source working group. Yearb. Med. Inform. 27, 041-047 (2018).

59. Celi, L. A., Citi, L., Ghassemi, M. \& Pollard, T. J. The PLOS ONE collection on machine learning in health and biomedicine: Towards open code and open data. PLoS ONE 14, e0210232 (2019).

60. Morris, J. C. The clinical dementia rating (CDR): Current version and scoring rules. Neurology 43, 2412-2414 (1993)

61. Morris, J. C. et al. Mild cognitive impairment represents early-stage Alzheimer disease. Arch. Neurol. 58, 397-405 (2001)

62. Hoehn, M. M. \& Yahr, M. D. Parkinsonism: Onset, progression and mortality. Neurology 17, 427-442 (1967).

63. Tessa, C. et al. Central modulation of parasympathetic outflow is impaired in de novo Parkinson's disease patients. PLoS ONE 14, $\mathrm{e} 0210324$ (2019).

64. Han, X. et al. Brain extraction from normal and pathological images: A joint PCA/image-reconstruction approach. Neuroimage 176, 431-445 (2018).

65. Avants, B. B. et al. A reproducible evaluation of ANTs similarity metric performance in brain image registration. Neuroimage 54, 2033-2044 (2011).

66. Bradski, G. R. \& Kaehler, A. Learning OpenCV: Computer Vision with the OpenCV Library (O’Reilly, 2011).

67. Simonyan, K. \& Zisserman, A. Very deep convolutional networks for large-scale image recognition. Preprint at http://arXiv.org/ 1409.1556 (2015). 
68. He, K., Zhang, X., Ren, S. \& Sun, J. Delving deep into rectifiers: Surpassing human-level performance on imagenet classification. In 2015 IEEE International Conference on Computer Vision (ICCV), 1026-1034. https://doi.org/10.1109/ICCV.2015.123 (IEEE, 2015).

69. Glorot, X. \& Bengio, Y. Understanding the difficulty of training deep feedforward neural networks. In Proc. Thirteenth International Conference on Artificial Intelligence and Statistics, 249-256 (2010).

70. Lin, M., Chen, Q. \& Yan, S. Network in network. Preprint at http://arXiv.org/1312.4400 (2014).

71. Szegedy, C. et al. Going deeper with convolutions. In 2015 IEEE Conference on Computer Vision and Pattern Recognition (CVPR), 1-9. https://doi.org/10.1109/CVPR.2015.7298594 (2015)

72. Hastie, T., Tibshirani, R. \& Friedman, J. H. The Elements of Statistical Learning: Data Mining, Inference, and Prediction (Springer, 2009).

73. Lemm, S., Blankertz, B., Dickhaus, T. \& Müller, K.-R. Introduction to machine learning for brain imaging. Neuroimage 56, 387-399 (2011).

74. Kingma, D. P. \& Ba, J. Adam: A Method for Stochastic Optimization. In CoRR (2015).

75. Cook, S. CUDA Programming: A Developer's Guide to Parallel Computing with GPUs (Elsevier Science, 2014).

76. TensorFlow Developers. TensorFlow (2021).

77. Chollet, F. Keras: The python deep learning library. ascl-1806 (Astrophysics Source Code Library, 2018).

78. Pedregosa, F. et al. Scikit-learn: Machine learning in python. J. Mach. Learn. Res. 12, 2825-2830 (2011).

79. Brett, M. et al. Nipy/nibabel: 2.3.3 (2019)

\section{Acknowledgements}

Data used in the preparation of this article were obtained from the Alzheimer's Disease Neuroimaging Initiative (ADNI) database (adni.loni.usc.edu). As such, the investigators within the ADNI contributed to the design and implementation of ADNI and/or provided data but did not participate in the analysis or writing of this report. A complete listing of ADNI investigators can be found at: http://adni.loni.usc.edu/wp-ontent/uploads/how_to_ apply/ADNI_Acknowledgement_List.pdf. Part of the data collection and sharing for this project was funded by the Alzheimer's Disease Neuroimaging Initiative (ADNI) (National Institutes of Health Grant U01 AG024904) and DOD ADNI (Department of Defense award number W81XWH-12-2-0012). ADNI is funded by the National Institute on Aging, the National Institute of Biomedical Imaging and Bioengineering, and through generous contributions from the following: AbbVie, Alzheimer's Association; Alzheimer's Drug Discovery Foundation; Araclon Biotech; BioClinica, Inc.; Biogen; Bristol-Myers Squibb Company; CereSpir, Inc.; Cogstate; Eisai Inc.; Elan Pharmaceuticals, Inc.; Eli Lilly and Company; EuroImmun; F. Hoffmann-La Roche Ltd and its affiliated company Genentech, Inc.; Fujirebio; GE Healthcare; IXICO Ltd.; Janssen Alzheimer Immunotherapy Research \& Development, LLC.; Johnson \& Johnson Pharmaceutical Research \& Development LLC.; Lumosity; Lundbeck; Merck \& Co., Inc.; Meso Scale Diagnostics, LLC.; NeuroRx Research; Neurotrack Technologies; Novartis Pharmaceuticals Corporation; Pfizer Inc.; Piramal Imaging; Servier; Takeda Pharmaceutical Company; and Transition Therapeutics. The Canadian Institutes of Health Research is providing funds to support ADNI clinical sites in Canada. Private sector contributions are facilitated by the Foundation for the National Institutes of Health (www. fnih.org). The grantee organization is the Northern California Institute for Research and Education, and the study is coordinated by the Alzheimer's Therapeutic Research Institute at the University of Southern California. ADNI data are disseminated by the Laboratory for Neuro Imaging at the University of Southern California. Data were provided [in part] by OASIS: Cross-Sectional: Principal Investigators: D. Marcus, R, Buckner, J, Csernansky J. Morris; P50 AG05681, P01 AG03991, P01 AG026276, R01 AG021910, P20 MH071616, U24 RR021382. Data used in the preparation of this article were [in part] obtained from the Parkinson's Progression Markers Initiative (PPMI) database www.ppmi-info.org/data. For up-to-date information on the study, visit www.ppmi-info.org. PPMI-a public-private partnership-is funded by the Michael J. Fox Foundation for Parkinson's Research and multiple funding partners. The full list of PPMI funding partners can be found at ppmi-info.org/fundingpartners.

\section{Author contributions}

E.Y., S.W.A., A.G., L.C. and S.D. conceived the original idea. C.M. and S.D. performed the data pre-processing. E.Y. and S.W.A developed the software implementation of the model training and validation. R.S. helped with the software implementation and creation of the Docker image. E.Y., S.W.A, A.G., C.M., L.C. and S.D. drafted the manuscript and designed figures and tables. All authors contributed to the interpretation of the results and revised the final version of the manuscript.

\section{Funding}

This work was supported in part by the European Union's Horizon 2020 research and innovation programme under Grant Agreement No 824153 "POTION" (EY and LC) and in part by an NVIDIA Academic GPU Grant Program (SD).

\section{Competing interests}

The authors declare no competing interests.

\section{Additional information}

Supplementary Information The online version contains supplementary material available at https://doi.org/ 10.1038/s41598-021-01681-w.

Correspondence and requests for materials should be addressed to S.D.

Reprints and permissions information is available at www.nature.com/reprints.

Publisher's note Springer Nature remains neutral with regard to jurisdictional claims in published maps and institutional affiliations. 
(c) (i) Open Access This article is licensed under a Creative Commons Attribution 4.0 International cc) License, which permits use, sharing, adaptation, distribution and reproduction in any medium or format, as long as you give appropriate credit to the original author(s) and the source, provide a link to the Creative Commons licence, and indicate if changes were made. The images or other third party material in this article are included in the article's Creative Commons licence, unless indicated otherwise in a credit line to the material. If material is not included in the article's Creative Commons licence and your intended use is not permitted by statutory regulation or exceeds the permitted use, you will need to obtain permission directly from the copyright holder. To view a copy of this licence, visit http://creativecommons.org/licenses/by/4.0/.

(C) The Author(s) 2021 